\title{
Buchbesprechungen
}

\section{Das Harnblasenkarzinom}

Epidemiologie, Pathogenese, Früherkennung

Herausgeber: K.-H. Bichler und R. Harzmann Springer, Berlin, Heidelberg, New York, Tokyo 1984237 Seiten, 129 Abb., DM 92-ISBN 3-540-13115-9

Die Beiträge dieses Buches resultieren aus einer interdiszipli-nären Arbeitstagung in Tubingen, bei der Aspekte der Prä-vention durch die Ausschaltung vermeidbarer Risiken sowie Möglichkeiten zur Verbesserung der Prognose durch Früh-erkennungsmaßnahmen im Vordergrund standen. Dement-sprechend findet sich zunächst ein Überblick von K. Norpoth zu den möglichen exogenen und endogenen Ursachen. G. Ru-tishauser et al. diskutieren über die Problematik des Analgeti-kaabusus und darüber, daß rund 16\% der Urothelkarzinome durch einen Phenacetinabusus induziert sind. R. Harzmann et al. zeigen, daß mit in-vitroTestverfahren, wie dem bakte-riellen Mutagenesetest (Salmonella nyphimurium), der Nach-weis von mutagenen bzw. karzinogenen Substanzen im Urin möglich ist und daß damit Risikosituationen erfaßt werden und zu Präventivmaßnahmen Anlaß geben können. Unter dem Aspekt der multifaktoriellen Mehrstufenkarzinogenese erörtert E. Kunze die Notwendigkeit, nicht nur nach potenten Solitärkarzinogenen zu fahnden, sondern verstärkt nach inkompletten Karzinogenen wie ko-karzinogenen bzw. pro-movierenden Faktoren, vor allem in der außerberuflichen Umwelt. G. E. Schubert demonstriert die Häufigkeit prolifera-tiver Urothelveränderungen bei Männern und Frauen mit einem mittleren Alter von 73 Jahren und erörtert ihre prä-maligne Bedeutung. St. Peter erläutert, daß ultrastrukturelle Membranveränderungen zur ungeordneten Zellteilung und zu invasivem Wachstum führen können. Der Verbesserung von Screening, Früherkennung, Grading und Verlaufskontrolle soil die Automatisierung der Urinzytologie dienen, sei es durch die Beurteilung nukleärer Merkmale wie DNS- und Chromatinkontrast in einem Bildanalysesystem (H.J.Tanke et al.), sei es durch impulszytophotometrische DNS-Bestim-mungen (A. Zimmermann) oder durch die Kombination von zytologischen, impulszytophotometrischen und zellkinetischen Untersuchungen (Helpap et al.). Besondere Aufmerksamkeit verdienen in diesem Zusammenhang auch immunzytologische bzw. immunhistochemische Untersuchungen bezüglich des Verhaltens der Blutgruppenantigene (H.J. Nelde und K.-H. Bichler sowie G. Jakse und F. Hofstädter). H.-D. Adolphs und P. Oehr demonstrieren den Vorteil der alleinigen TPA-Bestim-mung im Serum und Urin gegenüber der CEA-Bestimmung bzw. gegenüber Markerkombinationen. Offen ist die Bedeutung von Glykosaminoglykanen, die K.-H.Bichler und S. Korn bei Patienten mit Harnblasenkarzinomen im Urin vermindert fanden und die H.Rübhen et al. erstmals durch eine biochemische Analyse im Urothel und in Urothelkarzino-

men qualitativ und quantitativ bestimmten. R. Tauber und D.Jüngst fanden bei Patienten mit Harnblasentumoren in Abhängigkeit vom klinischen Stadium deutliche Erhöhungen der Cholesterinausscheidung und wiesen an Patienten mit Mikrohämaturien nach, daß die Cholesterinbestimmung zur besseren Erfassung einer tumorbedingten Mikrohämaturie beitragen kann. Harzmann et al. stellten fest, daß die Serum-Hyaluronidase mit dem Grad der 
Entdifferenzierung erhöhte Werte aufweist, während sämtliche anderen Enzyme im Serum und Urin keine Markerqualitäten haben. Abschließend erörtert H. W. Bauer, daß die

Tumorimmunologie in der Dia-gnostik des Harnblasenkarzinoms noch im Stadium des klinischen Experimentes ist und J. E.Altwein demonstriert, daß instrumentelle Verfahren für die Früherkennung und für den Einsatz beim Massenscreening ungeeignet sind. Zu jedem Beitrag findet sich ein umfängliches Verzeichnis weiterführender Literatur, so daß das Buch einen aktuellen und kompletten Überblick bietet und jedem am Thema Inter-essierten zur Lektüre zu empfehlen ist.

W. Vahlensieck, Bonn

Aktuelle chirurgische Onkologie

Herausgeber: A. Encke, K. H. Jungbluth, H.-D. Röher und

M. Trede

Springer, Berlin, Heidelberg, New York 1982

82Abb., 88Tab., XI, 228 S., geb. DM 68,-; approx. US\$30.20

ISBN 3-540-11411-4

Der 70. Geburtstag von Prof. Dr. Dr. med. h.c. mult. F. Lindner, war der Anlaß für seine Schüler und Freunde, die vorliegenden Referate unter dem Titel: »Aktuelle chirurgische Onkologie« in einer Festschrift zusammenzufassen. Hierbei bietet die vorliegende Monographie eine gut gegliederte Darstellung des aktuellen Standes der chirurgischen Onkologie. In insgesamt 21 Beitragen werden chirurgisch-onkologische Behandlungskonzepte dargestellt und diskutiert. Dem Leser wird es im Einzelnen ermöglicht, durch jeweilige ausführliche Literaturverzeichnisse seine Kenntnisse in der dargestellten Problematik zu vertiefen. Hauptschwergewicht liegt in der Darstellung der chirurgischen Onkologie aus der Sicht des Allgemeinchirurgen, insbesondere über Krebsfor-men des Gastrointestinaltraktes, der Mamma sowie der endo-krinen Drüsen. Durch Einzelbeiträge werden auch onkologi-sche Probleme aus der Sicht des Thoraxchirurgen, des Uro-logen und Unfall- und Kinderchirurgen beleuchtet. In der vorliegenden praxisnahen Ausführung kann die Monographie jedem Leser, der sich kurz über ein onkologisches Problem aus chirurgischer Sicht informieren will, empfohlen werden.

H. Bindewald, Heidelberg 